\title{
Formation of Deeply Bound Pionic Atoms and Pion Properties in Nuclei
}

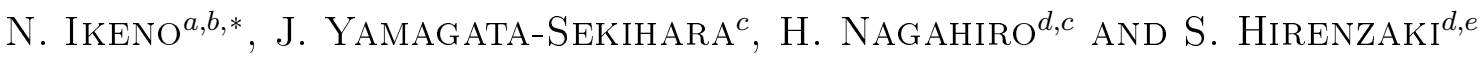 \\ ${ }^{a}$ Department of Physics, Tohoku University, Sendai 980-8578, Japan \\ ${ }^{b}$ YITP, Kyoto University, Kyoto 606-8502, Japan \\ ${ }^{c}$ RCNP, Osaka University, Ibaraki, Osaka 567-0047, Japan \\ ${ }^{d}$ Department of Physics, Nara Women's University, Nara 630-8506, Japan \\ ${ }^{e}$ J-PARC Branch, KEK Theory Center, Ibaraki 319-1106, Japan
}

\begin{abstract}
We study theoretically the formation of deeply bound pionic atoms to deduce the pion properties in nuclei precisely from the observables. We show the theoretical formation spectra of deeply bound pionic atoms in the various cases. We consider the pionic atom formation on the even-even and neutron-odd nucleus targets. We also show the angular dependence of the formation spectra in the $\left(d,{ }^{3} \mathrm{He}\right)$ reactions. Based on these theoretical results, we have found that we can obtain the systematic information on several deeply bound states for various nuclei. Actually, these observations have been performed in the experiments at RIBF/RIKEN.
\end{abstract}

DOI: $10.12693 /$ APhysPolA.127.1568

PACS: 21.85.+d, 21.65.-f, 21.65.Jk

\section{Introduction}

To know the hadron properties in the extreme conditions with high density and/or temperature is quite interesting in the contemporary hadron-nuclear physics since they provide valuable information on the aspects of the symmetry of strong interaction at finite density [1]. One of the good systems to observe in-medium hadron properties is the deeply bound pionic atom, which is a $\pi^{-}$atomic bound state hardly observed by X-ray spectroscopy, such as the $1 s$ or $2 p$ states in heavy nuclei [2]. The deeply bound pionic states have been experimentally produced in the $\left(d,{ }^{3} \mathrm{He}\right)$ reactions with the $\mathrm{Pb}$ and $\mathrm{Sn}$ targets by following theoretical predictions. In Ref. [3], the energy shifts and widths of the pionic $1 s$ state have been measured in three $\mathrm{Sn}$ isotopes. From these observations, the partial restoration of chiral symmetry is concluded by determining the pion weak decay constant $f_{\pi}$ using the Tomozawa-Weinberg and Gell-Mann-OakesRenner relations. However, to develop further studies of the pion properties and the chiral symmetry restoration pattern in nuclei, we need to obtain more accurate and systematic information on deeply bound pionic states, which are required to determine the pion-nucleus interaction uniquely and to fix the potential strength related to chiral symmetry for various cases beyond the linear density approximation.

We have developed further theoretical studies of the formation spectra of the pionic atoms in order to find out the appropriate procedure to obtain more precise information on chiral symmetry restoration from the observables [4-7]. New experiment at RIBF/RIKEN was

* corresponding author; e-mail: jan_ikeno@cc.nara-wu.ac.jp also performed and the observed spectra will be reported in near future $[8,9]$. In this article, we report the latest theoretical studies of the $\left(d,{ }^{3} \mathrm{He}\right)$ reactions for the pionic atom formation.

\section{2. $\left(d,{ }^{3} \mathbf{H e}\right)$ reaction on ${ }^{122} \mathrm{Sn}$ and ${ }^{117} \mathrm{Sn}$ targets}

We consider the neutron-odd nucleus ${ }^{117} \mathrm{Sn}$ as a target nucleus of the formation reaction in addition to the eveneven nucleus ${ }^{122} \mathrm{Sn}[5,6]$.

In the $\left(d,{ }^{3} \mathrm{He}\right)$ reaction for the pionic atom formation on an even-neutron nuclear target, one neutron in the target nucleus is picked-up and removed from the nucleus. For the even-neutron nuclear target cases, since the final pionic states are the pion-particle plus neutron-hole $\left[\pi \otimes \mathrm{n}^{-1}\right]$ states, we will have to take into account the residual interaction effects $[10,11]$ to deduce the pionnucleus interaction precisely from the high precision experimental data.

On the other hand, for the odd-neutron nuclear target cases, we expect to observe the pionic states with the ground state of the even-even nucleus with $J^{P}=0^{+}$, which will not be affected by the additional shifts due to the residual interaction effects. The observation of pionic states free from these effects is very important to obtain more accurate information on pion properties in the nuclei from data.

We study theoretically the $\left(d,{ }^{3} \mathrm{He}\right)$ spectra for the pionic atom formation using the effective number approach. In Figs. 1 and 2 we show the calculated formation spectra of the deeply bound pionic atoms in the ${ }^{117} \mathrm{Sn}\left(d,{ }^{3} \mathrm{He}\right)$ and ${ }^{122} \mathrm{Sn}\left(d,{ }^{3} \mathrm{He}\right)$ reactions at the forward angle $\theta_{d \mathrm{He}}^{\text {lab }}=0^{\circ}$ and at the finite angle $\theta_{d \mathrm{He}}^{\text {lab }}=2^{\circ}$. In both the ${ }^{122} \mathrm{Sn}\left(d,{ }^{3} \mathrm{He}\right)$ and ${ }^{117} \mathrm{Sn}\left(d,{ }^{3} \mathrm{He}\right)$ spectra at the forward angle $\theta_{d \mathrm{He}}^{\mathrm{lab}}=0^{\circ}$, the contribution from the pionic $1 s$ state formation with picking-up one neutron from the $s_{1 / 2}$ state is found to be large because of the matching condition with the recoilless kinematics. 

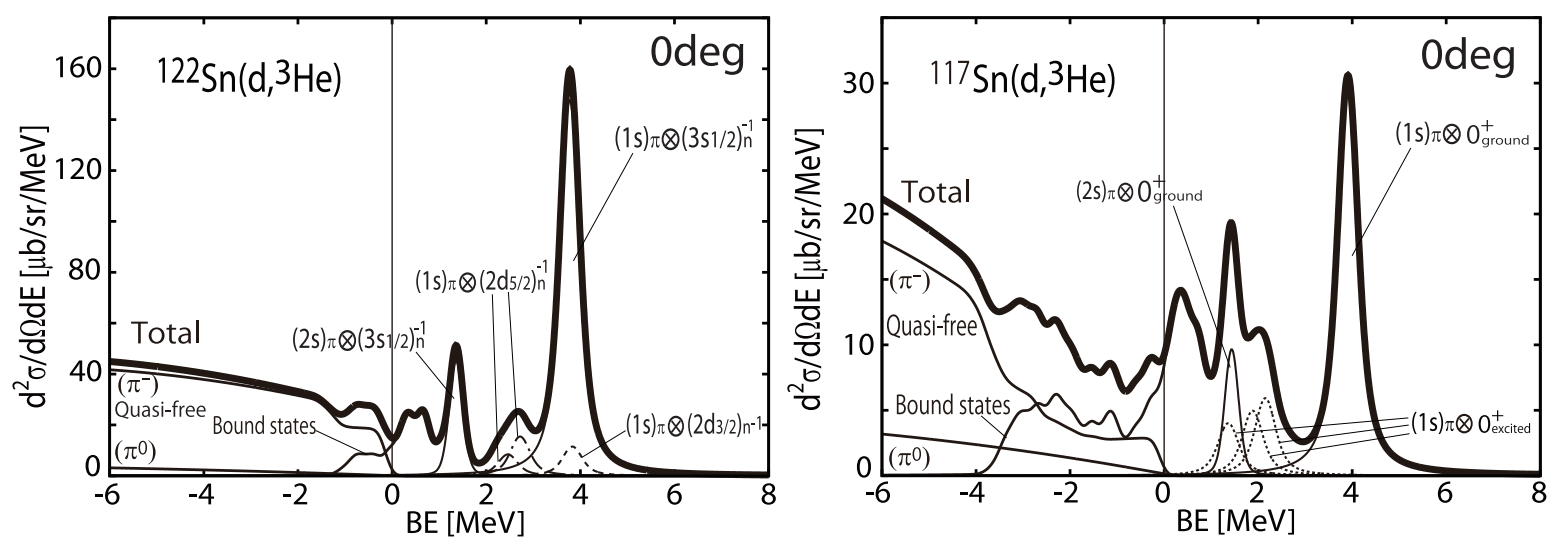

Fig. 1. Calculated spectra for the formation of the pionic states at $\theta_{d \mathrm{He}}^{\text {lab }}=0^{\circ}$ in the ${ }^{122} \operatorname{Sn}\left(d,{ }^{3} \mathrm{He}\right)$ (left) and the ${ }^{117} \mathrm{Sn}\left(d,{ }^{3} \mathrm{He}\right)$ (right) reactions plotted as functions of the pion binding energy reported in Ref. [6]. The incident deuteron kinetic energy is fixed to be $T_{d}=500 \mathrm{MeV}$. The total spectra are shown by the thick solid lines in both figures. We show separately the contributions of the bound pionic states formation and the quasi-free $\pi^{-}$and $\pi^{0}$ production by the thin solid lines. The dominant subcomponents are also shown in the figures with quantum numbers indicated as $\left[(\mathrm{n} \ell)_{\pi} \otimes\left(\mathrm{n} \ell_{j}\right)_{\mathrm{n}}^{-1}\right]$ in the ${ }^{122} \mathrm{Sn}\left(d,{ }^{3} \mathrm{He}\right)$ reaction and $\left[(\mathrm{n} \ell)_{\pi} \otimes J^{P}\right]$ in the ${ }^{117} \mathrm{Sn}\left(d,{ }^{3} \mathrm{He}\right)$ reaction, respectively. The instrumental energy resolution is assumed to be $300 \mathrm{keV}$ FWHM.
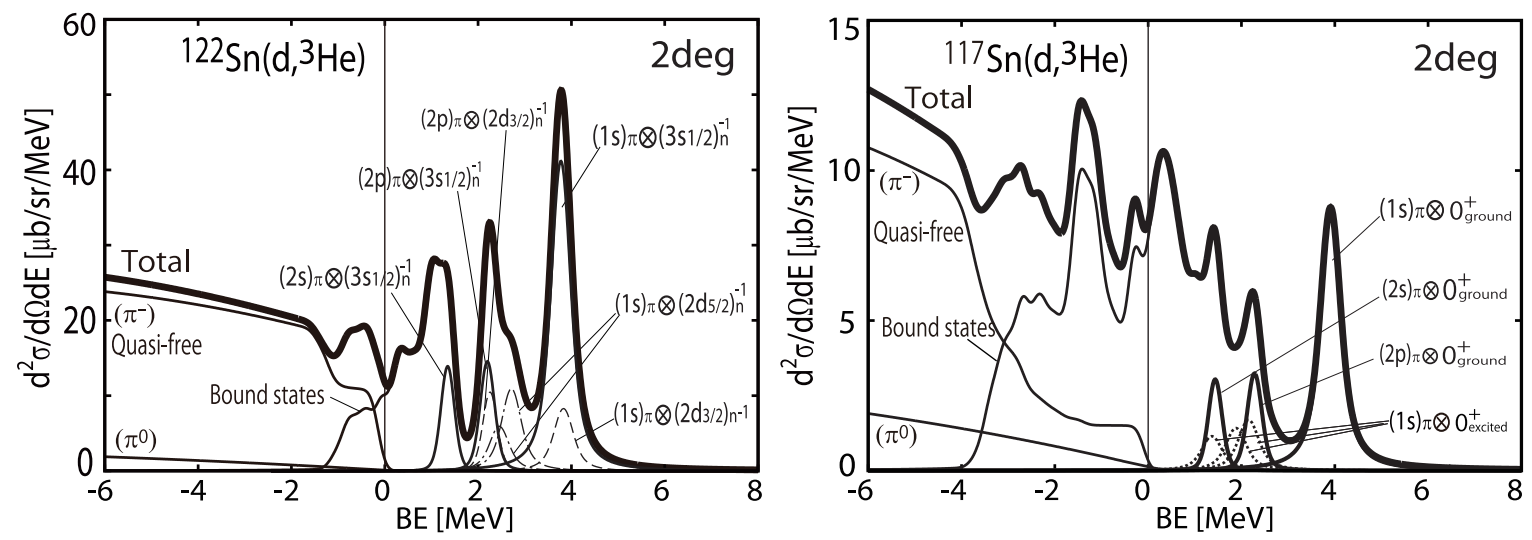

Fig. 2. Calculated spectra for the formation of the pionic states at $\theta_{d \mathrm{He}}^{\text {lab }}=2^{\circ}$ in the ${ }^{122} \operatorname{Sn}\left(d,{ }^{3} \mathrm{He}\right)$ (left) and the ${ }^{117} \mathrm{Sn}\left(d,{ }^{3} \mathrm{He}\right)$ (right) reactions plotted as functions of the pion binding energy. These results are obtained by the same theoretical model described in Ref. [6]. The incident deuteron kinetic energy is fixed to be $T_{d}=500 \mathrm{MeV}$. The instrumental energy resolution is assumed to be $300 \mathrm{keV}$ FWHM.

At larger angles $\theta_{d \mathrm{He}}^{\text {lab }}=2^{\circ}$, we find that the ${ }^{122} \mathrm{Sn}\left(d,{ }^{3} \mathrm{He}\right)$ spectra are dominated by the subcomponents including $(2 p)_{\pi}$ state, while they are dominated by the $(1 s)_{\pi}$ and $(2 s)_{\pi}$ states in forward angles. Thus, we can conclude that we obtain information on the deeply bound pionic $2 p$ state in addition to the $1 s$ and $2 s$ states by observing the spectra in finite angles $\theta_{d \mathrm{He}}^{\text {lab }}=0^{\circ}$. As indicated in Ref. [4], the observation of several deeply pionic bound states in a certain nucleus will help to deduce precise information on the pion properties and the chiral dynamics at finite density.

We find that the ${ }^{117} \mathrm{Sn}\left(d,{ }^{3} \mathrm{He}\right)$ spectra show the isolated peak structure with the single subcomponent of the pionic $1 s$ state formation with the ground state of the even-even nucleus $\left(\left[(1 s)_{\pi} \otimes 0_{\text {ground }}^{+}\right]\right)$, which has no residual interaction effects. Thus, the formation of this pionic $1 s$ state is preferable to extract the most accurate in- formation on the parameter of the QCD symmetry from the observation. We can say that the spectrum of the ${ }^{117} \mathrm{Sn}\left(d,{ }^{3} \mathrm{He}\right)$ reaction is more suited for the observation of the single subcomponent than that of the ${ }^{122} \mathrm{Sn}\left(d,{ }^{3} \mathrm{He}\right)$ reaction.

\section{Summary}

We have reported the recent theoretical studies of the formation of deeply bound pionic atoms. We have showed the ${ }^{117} \mathrm{Sn}\left(d,{ }^{3} \mathrm{He}\right)$ and ${ }^{122} \mathrm{Sn}\left(d,{ }^{3} \mathrm{He}\right)$ spectra at $\theta_{d \mathrm{He}}^{\text {lab }}=0^{\circ}$ and $\theta_{d \mathrm{He}}^{\mathrm{lab}}=2^{\circ}$. Recently, the experiment for the pionic atom formation on ${ }^{117} \mathrm{Sn}$ and ${ }^{122} \mathrm{Sn}$ nuclear targets have been performed at RIBF/RIKEN $[8,9]$. We believe that our studies will provide the systematic information on the pionic bound states in various nuclei and will help to develop the study of the pion properties and the partial restoration of chiral symmetry in nuclei. 


\section{References}

[1] T. Hatsuda, T. Kunihiro, Phys. Rep. 247, 221 (1994).

[2] T. Yamazaki, S. Hirenzaki, R.S. Hayano, H. Toki, Phys. Rep. 514, 1 (2012).

[3] K. Suzuki, M. Fujita, H. Geissel, H. Gilg, A. Gillitzer, R. S. Hayano, S. Hirenzaki, K. Itahashi, M. Iwasaki, P. Kienle, M. Matos, G. Münzenberg, T. Ohtsubo, M. Sato, M. Shindo, T. Suzuki, H. Weick, M. Winkler, T. Yamazaki, T. Yoneyama, Phys. Rev. Lett. 92, 072302 (2004).

[4] N. Ikeno, R. Kimura, J. Yamagata-Sekihara, H. Nagahiro, D. Jido, K. Itahashi, L.S. Geng, S. Hirenzaki, Prog. Theor. Phys. 126, 483 (2011).

[5] N. Ikeno, H. Nagahiro, S. Hirenzaki, Eur. Phys. J. A 47, $161(2011)$
[6] N. Ikeno, J. Yamagata-Sekihara, H. Nagahiro, S. Hirenzaki, Prog. Theor. Exp. Phys. 2013, 063D01 (2013).

[7] N. Ikeno, J. Yamagata-Sekihara, H. Nagahiro, S. Hirenzaki, Prog. Theor. Exp. Phys. 2015, 033D01 (2015).

[8] K. Itahashi, presentation on Int. Conf. on Exotic Atoms and Related Topics (EXA2014), 2014, Vienna (Austria).

[9] K. Itahashi, T. Nishi, private communication.

[10] S. Hirenzaki, H. Kaneyasu, K. Kume, H. Toki, Y. Umemoto, Phys. Rev. C 60, 058202 (1999).

[11] N. Nose-Togawa, H. Nagahiro, S. Hirenzaki, K. Kume, Phys. Rev. C 71, 061601(R) (2005). 